\title{
Variational mean-field study of a continuum model of crystalline tensionless surfaces
}

\author{
Esteban Moro ${ }^{1,2}$ and Rodolfo Cuerno ${ }^{2}$ \\ ${ }^{1}$ Theoretical Physics, University of Oxford, 1 Keble Road, Oxford OX1 3NP, United Kingdom \\ ${ }^{2}$ Departamento de Matemáticas and Grupo Interdisciplinar de Sistemas Complicados, Universidad Carlos III de Madrid, \\ Avda. Universidad 30, E-28911 Leganés, Spain
}

\begin{abstract}
We study analytically the equilibrium and near-equilibrium properties of a model of a $d$-dimensional surface relaxing via linear surface diffusion and subject to a lattice potential. We employ the variational mean-field formalism introduced by Saito for the study of the sine-Gordon model. In equilibrium, our variational theory predicts a first-order roughening transition between a flat low-temperature phase and a rough high-temperature phase with the properties of the linear molecular-beam epitaxy equation. Moreover, the study of a Gaussian approximation to the Langevin dynamics of the system indicates that the surface shows hysteresis when temperature is continuously tuned. Out of equilibrium, these approximate Langevin dynamics show that the surface mobility can have different behaviors as a function of a driving flux. Some considerations are made regarding different underlying lattices, and connections are drawn to related models or different approaches to the same model we study.
\end{abstract}

DOI: 10.1103/PhysRevE.63.036104

PACS number(s): 64.60.Ht, 64.60.Cn, 68.35.Rh, 81.10.Aj

\section{INTRODUCTION}

During the last decade, there have been great theoretical and experimental efforts to understand surface growth. This is due to possible applications, e.g., to the production of thin films and, from the basic point of view, to the interesting examples growing surfaces provide of nonequilibrium statistical systems [1], in some cases with strong relation to relevant equilibrium systems [2]. A very important example is provided by the discrete Gaussian (dG) model, which describes the universal features of the equilibrium roughening transition of many surfaces [3]. This transition is driven by temperature fluctuations, the value of the critical temperature being nowadays regarded as a standard property of, e.g., metals [4]. The equilibrium roughening transition of the dG model is in the Kosterlitz-Thouless (KT) class, and thus the model is related to other important models featuring a similar transition, such as the $F$ model or the Coulomb gas $[2,3]$. The dG model describes a surface minimizing surface area (to linear approximation), in which the surface height takes on integer values. Relaxing the latter condition leads to the sine-Gordon ( $\mathrm{sG}$ ) model for a real valued height field subject to surface tension and to a (lattice) potential favoring integer values of the field. The $\mathrm{sG}$ model is amenable to approximate analytic treatments $[5,6]$, which have allowed to develop a rather complete picture of the equilibrium roughening transition, and of the near-equilibrium properties as determined by Langevin dynamics [7] or kinetic Monte Carlo simulations [8]. However, several authors have recently raised the idea that the KT transition in the $\mathrm{sG}$ model might be replaced by some kind of first-order discontinuity $[9,10]$ when the strength of the potential is increased further away from the renormalization-group ( $R G)$ perturbative regime, a result whose existence had been speculated for a long time [11]. In this strong potential regime, the perturbative RG treatments break down and mean-field-type methods have been used to study the transition.

There exist some interface phenomena contexts in which the most relevant relaxation mechanism taking place at the interface can be modeled as minimization of surface curvature, instead of minimization of surface area as in the $\mathrm{dG}$ model. Instances of these phenomena are membranes [12], two-dimensional melting [13], or growth by molecular-beam epitaxy (MBE) [1] in which the main relaxation mechanism is surface diffusion, which in the simplest approximation is described effectively as surface curvature minimization. In a close analogy with the dG model, several authors have studied the effect of the integer height value restriction in these situations, but the understanding is not as complete as in the $\mathrm{dG}$ case. For instance, in some investigations of the twodimensional melting problem [13] Nelson was led to formulate the discrete Laplacian roughening model $(\mathrm{dLr})$ model

$$
\mathcal{H}=\frac{\kappa}{2} \sum_{\mathbf{r}}[\Delta h(\mathbf{r})]^{2}, \quad h(\mathbf{r}) / a_{\perp} \in \mathbb{Z}
$$

where $\mathbf{r}$ is the lattice position on a $L \times L$ dimensional substrate, $a_{\perp}$ is the vertical lattice constant, and $\Delta$ is the lattice approximation to the Laplacian operator. This paper indicated that Hamiltonian (1) should have two phase transitions, both of them in the KT universality class, which are related with the unbinding of disclinations and dislocations in the melting problem. This two-step melting mechanism is known as the Kosterlitz-Thouless-Nelson-Halperin-Young theory for two-dimensional melting. Despite the analytical and numerical efforts spent [14], this picture has not been fully verified for model (1), some authors claiming a single first-order roughening transition occurs [15-17]. The situation is not settled either for the actual physical problemnamely, two-dimensional melting-the Hamiltonian (1) was intended to model [18], since both first-order and KT-type transitions have been observed in experiments and numerical simulations.

On the other hand, in Ref. [19] the following Hamiltonian was proposed to study the effects of the crystalline structure in tensionless surfaces 


$$
\mathcal{H}=\frac{\kappa}{2} \sum_{\mathbf{r}}\left\{[\Delta h(\mathbf{r})]^{2}+V_{0}\left[1-\cos 2 \pi h(\mathbf{r}) / a_{\perp}\right]\right\},
$$

$$
h(\mathbf{r}) \in \mathrm{R} .
$$

Numerical Langevin simulations $[19,20]$ showed an equilibrium roughening transition, similar to that in the $\mathrm{sG}$ model. For temperatures below a critical value $T_{R}$, the lattice potential is relevant, imposing a finite value for the correlation length $\xi$, and thus the surface is flat. Namely, the roughness $w^{2} \equiv\left(1 / L^{2}\right) \Sigma_{\mathbf{r}}[h(\mathbf{r})-\bar{h}]^{2}\left[\right.$ where $\left.\bar{h}=\left(1 / L^{2}\right)\left\langle\Sigma_{\mathbf{r}} h(\mathbf{r})\right\rangle\right]$ is finite and $L$ independent. For temperatures higher that $T_{R}$, the lattice potential becomes irrelevant and $\xi$ diverges, the surface being rough. This means that the roughness diverges with the system size, in the same way as occurs if we take $V_{0} \equiv 0$ in Eq. (2), for which case the Langevin dynamics is that of the so-called linear MBE equation [1]. In studies of epitaxial growth systems by Langevin dynamics, the term proportional to $\kappa$ in Eq. (2) generates the relevant surface diffusion effect (within a linear approximation in the surface slopes), whereas the $V_{0}$ term models in the simplest way effects due to a crystalline structure. Larger numerical simulations of Eqs. (1) and (2) are nevertheless needed [21] in order to settle the question about the character of the equilibrium phase transition. On the other hand, Langevin simulations were extended to out-of-equilibrium situations $[19,20]$, finding that the mobility (to be defined below) behaves in various different ways, depending on the model parameters.

The above results make it even more natural to expect that both Hamiltonians (1) and (2) are related with each other in a similar way as the $\mathrm{dG}$ and $\mathrm{sG}$ models are. In fact, the Hamiltonian (2) can indeed be derived (see Appendix A) from Eq. (1) using certain approximations [22], although the derivation we present is not unique. Other authors have proposed different approximations (through a mapping to a vector Coulomb gas) to reproduce the integer height restriction [23], yielding continuum approximations of Eq. (1) different from Eq. (2). In this paper we restrict ourselves to the study of the properties of Eq. (2), in the hope that they will reflect some of the properties of the dLr model (1). Specifically, our aim is to study analytically the Lr model (1) using the continuum approximation (2) and a variational mean-field method proposed by Saito two decades ago [5] for the sG model. Despite its simplicity, this method anticipated the value of the transition temperature and the infinite-order character of the transition occurring in the sG model, which were later confirmed by renormalization-group calculations and by numerical simulations [24]. Since then, this variational mean-field theory has been used in other interface problems, like the preroughening transition [25], surface growth over disordered substrates [26], etc., showing that despite its simplicity this approach can indeed explain the topological properties of the corresponding phase diagram and, in some cases, provide accurate predictions for more specific properties like the values of the transition temperature and of the critical exponents. One might argue that in this mean-field-type treatment, fluctuations are not handled properly and might eventually modify the phase diagram Nevertheless, based on the success of Saito's mean-field treatment for the $\mathrm{sG}$ model, we expect the results of our analysis to be relevant to model (2), in particular for high dimensions, where fluctuations are suppressed.

The present paper is organized as follows. In Sec. II we study the equilibrium Hamiltonian (2) for a two-dimensional substrate, within the variational scheme of Ref. [5]; we derive expressions for the roughening temperature and correlation length below the transition. The precise value of the transition temperature within this approach is determined in the process of elucidating the character of the transition. Section III is devoted to the approximate study of the Langevin dynamics of (2) within a Gaussian approximation for the probability distribution of the height field. A discussion of the results obtained and our conclusions are found in Sec. IV. Appendix A contains the formal derivation of the continuum model (2) from Eq. (1). Some computational details on the solution of self-consistent equations relevant to Sec. II can be found in Appendix B, while Appendix C discusses how the results are modified when considering the model on a triangular lattice (as opposed to the square lattice studied in the rest of the paper), and Appendix D contains a discussion on results for substrate dimensions different from two.

\section{VARIATIONAL MEAN-FIELD METHOD: EQUILIBRIUM PROBLEM}

Following Saito [5], our main assumption is that the most relevant features of model (2) can be described by a simpler, solvable Hamiltonian:

$$
\mathcal{H}_{0}=\frac{T}{2} \sum_{\mathbf{q}} S^{-1}(\mathbf{q}) h(\mathbf{q}) h(-\mathbf{q})
$$

where $h(\mathbf{q})$ are the Fourier components of the height field

$$
h(\mathbf{q})=\frac{1}{L} \sum_{j} e^{i \mathbf{q} \cdot \mathbf{r}_{j}} h_{j}
$$

Here we consider periodic boundary conditions. Thus, $q_{x}$ $=2 \pi n_{x} / L$ with $n_{x}=-(L-1) / 2, \ldots, L / 2$ and a similar relation holds for $q_{y}$. Equation (3) defines a Gaussian Hamiltonian in which the values of $S(\mathbf{q})$ are $L^{2}$ free parameters. We will fix them by minimization of the variational free energy $\mathcal{F}_{V} \equiv \mathcal{F}_{0}+\left\langle\mathcal{H}-\mathcal{H}_{0}\right\rangle_{0}$, which is known to be an upper bound of the exact free energy $\mathcal{F}$ of model (2) by the Bogoliubov thermodynamic inequality [2]

$$
\mathcal{F} \leqslant \mathcal{F}_{V} \equiv \mathcal{F}_{0}+\left\langle\mathcal{H}-\mathcal{H}_{0}\right\rangle_{0}
$$

where $\mathcal{F}_{0}$ is the free energy of model $\mathcal{H}_{0}$ and $\langle\cdots\rangle_{0}$ stands for the average with respect to the Boltzmann factor $e^{-\mathcal{H}_{0} / T}$.

Using the Hamiltonians (2) and (3) we obtain for the right-hand side of Eq. (5): 


$$
\begin{aligned}
\frac{\mathcal{F}_{V}}{T}= & -\frac{1}{2} \sum_{\mathbf{q}} \ln 2 \pi S(\mathbf{q})+\frac{1}{2} \sum_{\mathbf{q}}\left[S_{0}^{-1}(\mathbf{q})-S^{-1}(\mathbf{q})\right] S(\mathbf{q}) \\
& +\frac{L^{2} V_{0}}{T}\left\{1-\exp \left(-\frac{2 \pi^{2}}{a_{\perp}^{2}} w^{2}\right)\right\}
\end{aligned}
$$

where we have defined $S_{0}=T /[\kappa \omega(\mathbf{q})]$ with $\omega(\mathbf{q})$ $=16\left[\sin ^{2}\left(q_{x} / 2\right)+\sin ^{2}\left(q_{y} / 2\right)\right]^{2}$ and

$$
w^{2}=\frac{1}{L^{2}} \sum_{j} h_{j}^{2}=\frac{1}{L^{2}} \sum_{\mathbf{q} \neq 0} S(\mathbf{q})
$$

[note that model (2) is symmetric under $h \rightarrow-h$ and thus, in equilibrium, $\bar{h} \equiv 0]$. By minimizing $\mathcal{F}_{V}$ with respect to the parameters $S(\mathbf{q})$, we find they have to verify

$$
S^{-1}(\mathbf{q})=S_{0}^{-1}(\mathbf{q})+4 \pi^{2} \frac{V_{0}}{a_{\perp}^{2} T} \exp \left(-\frac{2 \pi^{2}}{a_{\perp}^{2}} w^{2}\right) .
$$

We can rewrite Eq. (8) by noting that the second term on the right-hand side does not depend on q. Hence

$$
S(\mathbf{q})=\frac{T}{\kappa\left[\omega(\mathbf{q})+\xi^{-4}\right]},
$$

where $\xi$ is a constant given by the self-consistent relation [note $w^{2}$ depends on $\xi$ through Eqs. (7) and (9)]

$$
\kappa \xi^{-4}=\frac{4 \pi^{2} V_{0}}{a_{\perp}^{2}} \exp \left(-\frac{2 \pi^{2}}{a_{\perp}^{2}} w^{2}\right) .
$$

Equations (9) and (10) are the solution to the equilibrium problem. We observe that the variational (Gaussian) approximation of Hamiltonian (2) has a structure factor $S(\mathbf{q})$ similar to that of the linear MBE equation. The only effect of the potential is to introduce a correlation length $\xi$ given selfconsistently by Eqs. (10) and (9). Among all mathematical solutions of Eq. (10), the best approximation to model (2) is given by that value of $\xi$ that minimizes the variational free energy $\mathcal{F}_{V}$, which we denote by $\xi_{\text {phys }}$. Note all roots of Eq. (10) can be easily shown to be stationary points of the function $\mathcal{F}_{V}(\xi)$.

In order to proceed analytically, we need to take the continuum limit of the integrals appearing in Eqs. (6) and (7). In this limit, we make the replacement $L^{-2} \Sigma_{\mathbf{q}} \rightarrow(2 \pi)^{-2} \int d \mathbf{q}$, and we can approximate $\omega(\mathbf{q})=\mathbf{q}^{4}$, hence using Eq. (9) we get

$$
w^{2} \simeq \frac{1}{(2 \pi)^{2}} \int d \mathbf{q} \frac{T}{\kappa\left[\omega(\mathbf{q})+\xi^{-4}\right]}=\frac{T \xi^{2}}{8 \kappa}-\frac{T}{4 \kappa \pi^{3}}+\mathcal{O}\left(\xi^{-4}\right) .
$$

Keeping the dominant term in the above equation (in powers of $\xi$ ), and defining $x=2 \kappa^{1 / 2} a_{\perp} T^{-1 / 2} \pi^{-1} \xi^{-1}$, Eq. (10) becomes

$$
x^{4}=\gamma e^{-1 / x^{2}},
$$

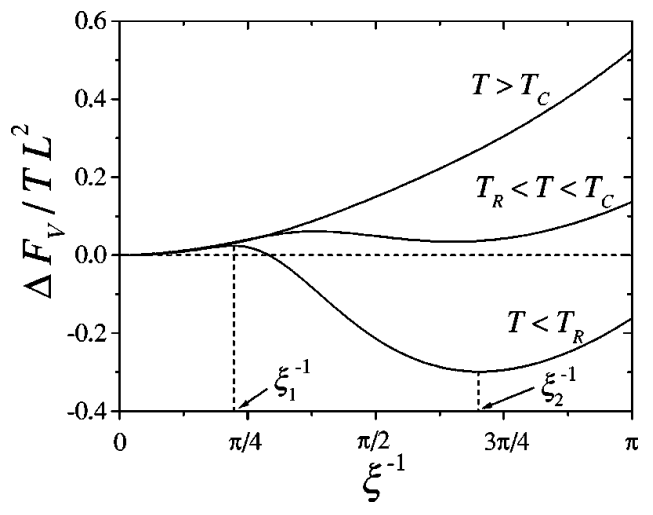

FIG. 1. Variational mean free-energy difference $\Delta \mathcal{F}_{V}$ as a function of the (inverse of the) correlation length for different temperatures. The values of $\xi_{1}$ and $\xi_{2}$ are only displayed for the $T<T_{R}$ case. The physical value of the correlation length $\xi_{\text {phys }}$ is given by the global minimum of $\Delta \mathcal{F}_{V}$. For temperatures $T>T_{R}$ the global minimum is always reached at $\xi^{-1}=0$. The parameters used are $V_{0}=a_{\perp}=\kappa=1$. Units are arbitrary.

where $\gamma=64 V_{0} a_{\perp}^{2} \kappa T^{-2} \pi^{-2}$. As shown in Appendix B, there are different solutions of Eq. (12) depending on $\gamma$ (and therefore on temperature). Thus, $\xi^{-1}=0$ is always a solution of Eq. (12), and is the unique solution for $T>T_{C}$ $=16 V_{0}^{1 / 2} \kappa^{1 / 2} a_{\perp} / e \pi$. However, for $T \leqslant T_{C}$ there appear two other finite solutions $0<\xi_{1}^{-1}<\xi_{2}^{-1}$ of Eq. (12). In order to check which of the three roots provides $\xi_{\text {phys }}$ in this temperature range, we compute the free-energy difference

$$
\begin{aligned}
& \frac{\Delta \mathcal{F}_{V}(\xi)}{T L^{2}} \equiv \frac{1}{T L^{2}}\left[\mathcal{F}_{V}(\xi)-\mathcal{F}_{V}\left(\xi^{-1}=0\right)\right] \\
& \simeq \frac{\xi^{-2}}{16}-\frac{V_{0}}{T} e^{-T \pi^{2} \xi^{2} /\left(4 \kappa a_{\perp}^{2}\right)}+\mathcal{O}\left(\xi^{-4}\right) .
\end{aligned}
$$

We plot $\Delta \mathcal{F}_{V}(\xi)$ in Fig. 1 for different values of $T$.

For $T \leqslant T_{C}$, as can be seen in the figure, $\Delta \mathcal{F}_{V}(\xi)$ has indeed a local maximum at $\xi_{1}^{-1}$ and a local minimum at $\xi_{2}^{-1}$, while for $T>T_{C}$ both disappear. As derived in Appendix B, for temperatures above $T_{R}=\left(e^{1 / 2} / 2\right) T_{C} \simeq 0.82 T_{C}$, the variational free-energy difference has its global minimum at $\xi_{\text {phys }}^{-1}=0$. However, for lower temperatures $T<T_{R}$, the finite correlation length $\xi_{2}$ features a lower value of the variational free energy than the infinite correlation length solution, hence $\xi_{\text {phys }}=\xi_{2}$ in this temperature range. Summarizing, within the variational approximation a roughening transition takes place at a temperature

$$
T_{R}=\frac{8}{\pi e^{1 / 2}} a_{\perp} \kappa^{1 / 2} V_{0}^{1 / 2}
$$

Above $T_{R}$ the correlation length is infinite and the surface is rough, with the same properties as the linear MBE model, i.e., $S(\mathbf{q}) \sim \mathbf{q}^{4}$ and $w^{2} \sim L^{2}$. Below $T_{R}$ the surface is flat with a finite correlation length equal to $\xi_{2}$. When we approach the 


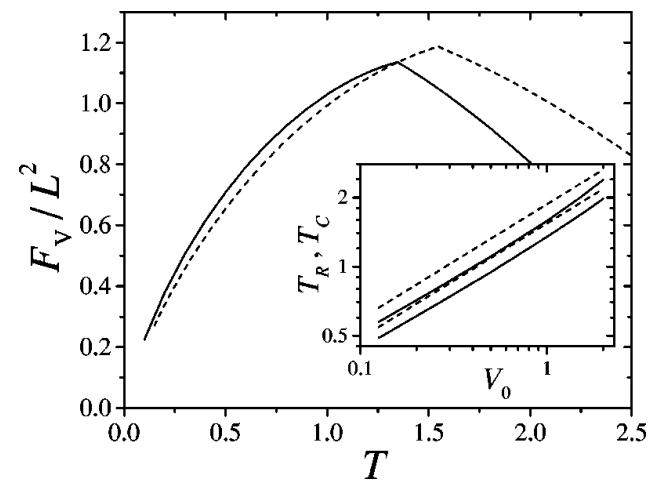

FIG. 2. Variational free energy $\mathcal{F}_{V}$ as a function of temperature for model (2) using the exact expression (6) (solid line) and our continuum approximation (dashed line). In both cases, $\mathcal{F}_{V}$ develops a cusp at $T=T_{R}$ due to the jump in the physical value of $\xi$. The inset shows the values of $T_{R}$ (lower curves) and $T_{C}$ as functions of $V_{0}$ within our continuum approximation (dashed lines) and using the exact discrete expressions (solid lines). In both cases, $T_{R} \propto T_{C}$ $\sim V_{0}^{1 / 2}$. The parameters used are $a_{\perp}=\kappa=1$ and the units are arbitrary.

roughening temperature from below, the correlation length does not diverge but, rather, tends to a constant value (see Appendix B) given by

$$
\xi\left(T \rightarrow T_{R}^{-}\right)=\left(\frac{4 \kappa a_{\perp}^{2}}{T_{R} \pi^{2}}\right)^{1 / 2}
$$

implying the roughening transition at $T_{R}$ is of first order. Specifically, a cusp develops in the free energy $\mathcal{F}_{V}$ as a function of temperature at $T=T_{R}$, as depicted in Fig. 2.

Although the results in this section have been obtained using a certain continuum approximation, we have numerically verified all our conclusions using the exact discrete sums in Eqs. (6) and (7). The exact variational results for the correlation length and the values of $T_{C}$ and $T_{R}$ for $L$ $=1024$ are compared in Fig. 2 to the approximate analytical expressions obtained in this section. We see that a first-order transition indeed takes place, although the values of $T_{R}$ and $T_{C}$ are modified. However, we still observe the nonlinear dependence of $T_{R}$ on $V_{0}$, see inset of Fig. 2.

\section{DYNAMICS WITHIN THE GAUSSIAN APPROXIMATION}

In this section, we study the near-equilibrium dynamics of model (2) by means of the generalized Langevin growth equation

$$
\frac{\partial h_{i}(t)}{\partial t}=F-\frac{\delta \mathcal{H}}{\delta h_{i}(t)}+\eta_{i}(t),
$$

where $\eta_{i}(t)$ is a white noise with correlations $\left\langle\eta_{i}(t) \eta_{j}\left(t^{\prime}\right)\right\rangle=2 T \delta_{i, j} \delta\left(t-t^{\prime}\right)$ and $F$ is the flux of incoming particles in the surface growth picture, or a chemicalpotential difference in a generic context. This equation describes not only the nonequilibrium statistical dynamics of our model, but also the dynamics of the system fluctuations around the equilibrium state (for $F=0$ ). Our approximation [5] to the study of Eq. (2) will be to assume a Gaussian time-dependent probability distribution for the height field. Thus, we only have to calculate the first two moments of the probability distribution, namely, the mean height [27] $\bar{h}$ $=\left\langle h_{i}(t)\right\rangle$ and the second moment $\langle h(\mathbf{q}, t) h(-\mathbf{q}, t)\rangle$ $=S(\mathbf{q}, t)$. Using Eqs. (2) and (17) (see a detailed account in Ref. [25]) we find

$$
\begin{aligned}
\frac{d \bar{h}}{d t}= & F-\frac{2 \pi V_{0}}{a_{\perp}}\left(\sin \left(\frac{2 \pi h_{i}}{a_{\perp}}\right)\right), \\
\frac{d S(\mathbf{q}, t)}{d t}= & -2 T S(\mathbf{q}, t)\left[S_{0}^{-1}(\mathbf{q})-S^{-1}(\mathbf{q}, t)\right. \\
& \left.+\frac{4 \pi^{2} V_{0}}{a_{\perp}^{2} T}\left(\cos \left(\frac{2 \pi h_{i}}{a_{\perp}}\right)\right)\right] \\
= & -4 T S(\mathbf{q}, t) \frac{\delta \mathcal{F}_{V}}{\delta S(\mathbf{q}, t)},
\end{aligned}
$$

where, within our Gaussian approximation,

$$
\begin{aligned}
& \left\langle\sin \left(\frac{2 \pi h}{a_{\perp}}\right)\right\rangle=e^{-2 \pi^{2} w^{2}(t) / a_{\perp}^{2} \sin \left(\frac{2 \pi \bar{h}}{a_{\perp}}\right),} \\
& \left\langle\cos \left(\frac{2 \pi h}{a_{\perp}}\right)\right\rangle=e^{-2 \pi^{2} w^{2}(t) / a_{\perp}^{2}} \cos \left(\frac{2 \pi \bar{h}}{a_{\perp}}\right)
\end{aligned}
$$

with $w^{2}(t)$ being the time-dependent surface roughness. In all cases, we will study the set of coupled differential equations (18) and (19) subject to the initial condition $h_{i}(t=0)$ $=0$ for all substrate positions $i$.

\section{A. Equilibrium}

In equilibrium, i.e., for $F=0$, the solution of Eq. (18) is $\bar{h}=0$ [note Eq. (21)] and the solution of Eq. (19) is the same as that of Eqs. (8) and (10) obtained in the previous section. The interest of Eq. (19) is that it allows us to study dynamically how the system chooses the physical value of the correlation length, and corroborate the results obtained in the previous section from the point of view of Langevin dynamics. Thus, we will integrate numerically the complete set of $L^{2}$ discrete equations (18) and (19) and perform the following experiment: starting from a flat surface and $T=0$, we increase temperature by a certain (small) amount and wait until the system reaches equilibrium. Then, we increase temperature by the same amount and repeat the equilibration process. When the temperature is high enough (i.e., once the system is in the rough phase) we decrease temperature by the same amount and repeat the process of equilibration until $T$ $=0$ is reached back closing a temperature cycle.

We observe that the equilibrium first-order transition found in the previous section indeed induces hysteresis in the system correlation length (see Fig. 3) when the system is 


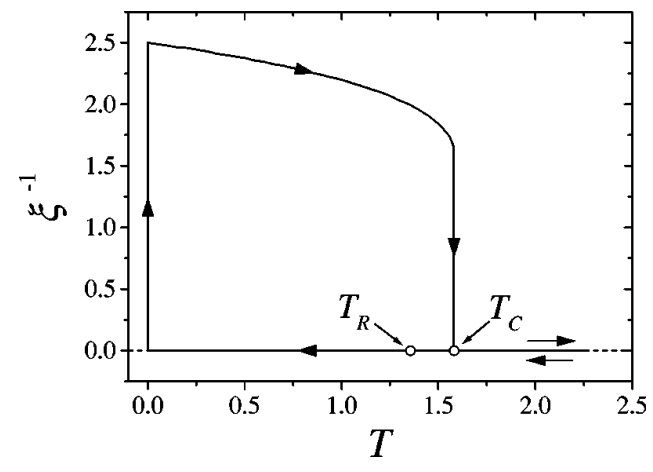

FIG. 3. (Inverse of the) physical correlation length as a function of temperature, as determined from Eqs. (19) and (9). The arrows indicate the heating and cooling experiment explained in the text. Parameters used are $V_{0}=a_{\perp}=\kappa=1$ and $L=1024$ and the units are arbitrary.

heated starting from $T=0$, in the sense that the roughening transition takes place at the higher temperature $T_{C}$ and not at $T_{R}$. The reason is that, for all $T$ up to $T_{C}$, the system stays in the local $\mathcal{F}_{V}$ minimum at $\xi_{2}$, even though for $T_{R}<T$ $<T_{C}$ the free energy already has its global minimum at $\xi^{-1}=0$, since there is an energy barrier for the system to jump across the local maximum in $\Delta \mathcal{F}_{V}$. Once the local minimum at $\xi_{2}$ disappears (i.e., for $T \geqslant T_{C}$ ), the surface is rough and exhibits an infinite correlation length. Conversely, when the system is cooled down starting at $T>T_{C}$, the system remains in the rough phase until $T=0$ is reached because $\xi^{-1}=0$ is always a free-energy minimum.

\section{B. Nonequilibrium}

In this section we allow $F \neq 0$ in Eqs. (18) and (19), in which case the former no longer has the trivial solution $(\bar{h}$ $=0$ ). Rather, when the flux $F$ is small (quasiequilibrium condition) we expect the system to feature a structure factor $S(\mathbf{q}, t)$ of the same form as in equilibrium, all nonequilibrium effects reflecting in the (possibly nontrivial) behavior of the average height. Actually, numerical simulations $[19,20]$ of the full nonlinear model (2) seem to confirm this expectation. For this reason we neglect the feedback effect of the evolution of $\bar{h}(t)$ on the structure factor $S(\mathbf{q}, t)$ and take

$$
S(\mathbf{q}, t) \simeq \frac{k_{B} T}{\kappa\left[\omega(\mathbf{q})+\xi^{-4}\right]},
$$

where $\xi$ is given by the physical equilibrium solution of Sec. II. Within this approximation,

$$
F_{c}(T) \equiv \frac{2 \pi V_{0}}{a_{\perp}} \exp \left\{-2 \pi^{2} w^{2} / a_{\perp}^{2}\right\}
$$

becomes a constant and Eq. (18) can be written as

$$
\frac{d \bar{h}}{d t}=F-F_{c} \sin \frac{2 \pi \bar{h}}{a_{\perp}}
$$

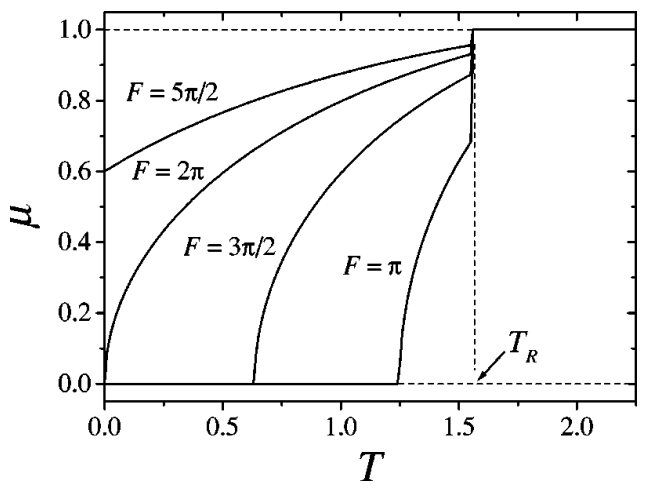

FIG. 4. Surface mobility as a function of $T$ for different values of the driving flux $F$. The values of the mobility are obtained from Eq. (25) using Eqs. (9) and (10) with the parameter values $V_{0}=\kappa$ $=a_{\perp}=1$ and the units are arbitrary.

which is simple to integrate analytically (exact expressions for the solution can be found in Refs. [5] and [25]). This equation has two different solutions depending on the values of $F$. If $F \leqslant F_{c}$, then $\bar{h}$ tends to a constant value and the surface does not grow. If we define the surface mobility $\mu$ as

$$
\mu=\frac{1}{F} \overline{\left\langle\frac{d \bar{h}}{d t}\right\rangle},
$$

where the overline stands for average over a time larger than $\mu^{-1}$, then for $F>F_{c}$, one obtains from the exact solution of Eq. (24) a nonzero value for $\mu$ :

$$
\mu=\left(1-\frac{F_{c}^{2}}{F^{2}}\right)^{1 / 2}
$$

In Fig. 4 we plot the surface mobility as a function of $T$.

Using the equilibrium solution for $\xi$ described in Sec. II, for temperatures above roughening $\left(T>T_{R}\right)$, we have that $\xi_{\text {phys }}^{-1}=0$, which implies $F_{c}=0$ and $\mu=1$. Thus, above roughening the surface shows linear growth with a maximum (unit) mobility. In the flat phase $\left(T<T_{R}\right)$ the mobility is equal to zero (i.e., the surface does not move) for a small flux $F<F_{c}(T)$. For larger values of the flux $\left[F>F_{c}(T)\right]$, the mobility depends nonlinearly on $T$ for all temperatures up to $T_{R}$ and actually the surface moves featuring an oscillatory roughness reminiscent of reflection high-energy electron diffraction oscillations in MBE systems, see Refs. [19,28]. Due to the jump of the correlation length at $T=T_{R}$, the mobility also has a jump at this temperature value. These three behaviors of the surface mobility as a function of temperature and driving flux agree with those obtained $[19,20]$ for the full model (2), except for the discrete jump of $\mu$ at $T=T_{R}$. They have been summarized in the $(T, F)$ phase diagram shown in Fig. 5, whose form differs from that computed numerically in Ref. [19] only in the mentioned jump of the mobility at $T=T_{R}$. A more detailed discussion on the relevance of Fig. 5 to, e.g., MBE systems can be found in Ref. [19]. 


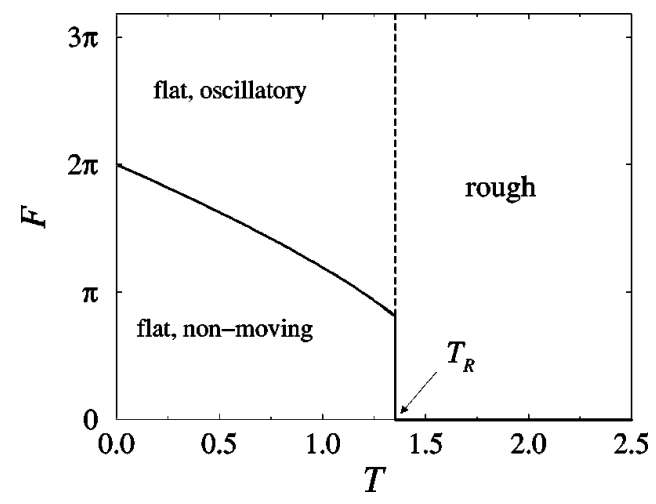

FIG. 5. Phase diagram as a function of temperature $T$ and driving flux $F$ for model (2) within the variational approximation. The solid line separating the flat, nonmoving and the flat, oscillatory phases is the locus $F=F_{c}(T)$. The parameters used are $V_{0}=a_{\perp}$ $=\kappa=1$ and the units are arbitrary.

\section{DISCUSSION AND CONCLUSIONS}

In this paper, we have performed an analytical approximation to the properties of model (2), which may be relevant to physical growth processes such as MBE or twodimensional melting. We have employed the variational approximation successfully applied by Saito to the study of the equilibrium roughening transition in the dG model. For our model system (2), the equilibrium results obtained in the previous sections predict a first-order phase transition and the associated hysteresis phenomenon. In particular, within a Gaussian approximation, Langevin dynamics predicts that a rough surface can preserve its infinite correlation length when cooled down across the roughening temperature. Moreover, we have found that these results apply both on the square and on the triangular lattices (see Appendix C). Hysteresis behavior and a first-order transition have been reported in Refs. [15] and [16] for models related with the dLr model (1) on the square lattice, and for the dLr model on the triangular lattice [17]. However, as mentioned in the Introduction, other authors seem to obtain two KT transitions for the $\mathrm{dLr}$ model, both on the square [29] and on the triangular [30] lattices. Note that our Langevin dynamics results within the Gaussian approximation yield a discrete jump in the surface mobility $\mu$ at $T=T_{R}$, which is not found in simulations of the full nonlinear model (2). This might indicate that the first-order character of the transition is in our case an artifact of the variational approximation. Moreover, this approximation (see Appendix D) also predicts a phase transition for model (2) in $d=1$, which is also obtained for the sG model. This result points out the limitations of this approximate scheme for situations in which fluctuations are very relevant for the system behavior, as in the $d=1$ case. Since model (2) features strong fluctuations (as does, e.g., the linear MBE equation [1]), it is desirable to go beyond our present meanfield approach to this model. We can take two steps in this direction. One (numerical) is to perform extended simulations of both the dLr model and model (2) [or, equivalently, its equilibrium Langevin dynamics (17)]. The results [21] seem to indicate that in both cases there is only one con- tinuum roughening transition as in the discrete Gaussian and sine-Gordon models, although with strong size dependence in the dLr case for sizes up to moderate, but not large. The other (analytical) improvement is to perform a dynamic RG analysis of Eq. (2) along the lines of that in Ref. [6] for the sG model. This study is particularly important bearing in mind that the lattice potential is expected to contribute a surface tension, absent in Eq. (2), which should then dominate the scaling behavior as compared with surface diffusion [1]. This phenomenon is clearly beyond our mean-field approach, which neglects parameter renormalization, and will be the subject of a forthcoming publication [31]. In any case, the study presented in this paper does indeed predict a phase diagram in two dimensions, including the numerical value of the roughening transition temperature, that compares well with numerical simulations $[20,21]$ of the complete model (2) and reflects the qualitative features of, e.g., the growth dynamics of some MBE systems. Moreover we expect the results presented here to be even more accurate in dimensions higher than two.

\section{ACKNOWLEDGMENTS}

The authors are pleased to thank Angel Sánchez for discussions, encouragement, and a detailed reading of the manuscript. This work was partially supported by DGES Grant Nos. PB96-0119 and HB1999-0018, and EPSRC Grant No. GR/M04426.

\section{APPENDIX A: DERIVATION OF THE CONTINUUM APPROXIMATION TO THE LAPLACIAN ROUGHENING MODEL}

Here we follow Ref. [22] in order to derive a continuum approximation to the dLr model (1). The dLr partition function is given by

$$
\mathcal{Z}_{\mathrm{dLr}} \equiv \sum_{\{h(\mathbf{r})\}} \exp \left\{-\frac{\kappa}{2 T} \sum_{\mathbf{r}}[\Delta h(\mathbf{r})]^{2}\right\}, \quad h(\mathbf{r}) / a_{\perp} \in \mathbb{Z}
$$

where the sum extends over all possible configurations of $h(\mathbf{r})$. The integer height condition can be implemented by using delta functions in the integrals, thus giving

$$
\begin{aligned}
\mathcal{Z}_{\mathrm{dLr}}= & \int \mathcal{D} h(\mathbf{r})\left[\sum_{n(\mathbf{r})=-\infty}^{\infty} \delta[h(\mathbf{r})-n(\mathbf{r})]\right] \\
& \times \exp \left\{-\frac{\kappa}{2 T} \sum_{\mathbf{r}}[\Delta h(\mathbf{r})]^{2}\right\},
\end{aligned}
$$

where $\mathcal{D} h(r) \equiv \Pi_{\mathbf{r}} d h(\mathbf{r})$. Inserting the following representation for the sum of delta functions in Eq. (A2),

$$
\sum_{n=-\infty}^{\infty} \delta(h-n)=\lim _{B \rightarrow \infty} \frac{B}{\sqrt{\pi}} \exp \left\{-B^{2} \sin ^{2} \pi h\right\}
$$

we obtain 


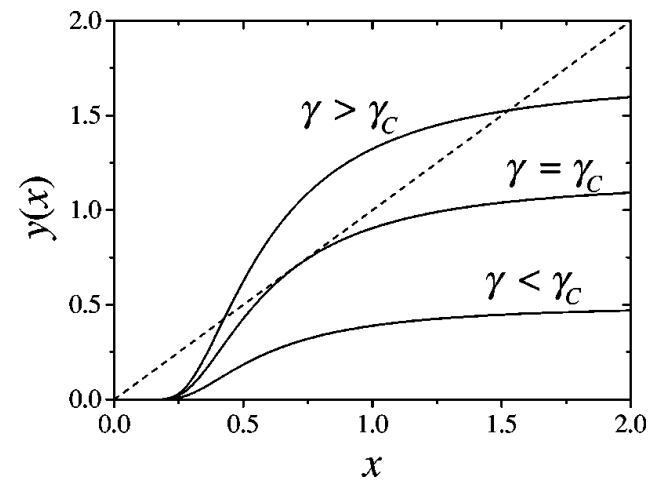

FIG. 6. Graphical representation of Eq. (B2). The dashed line is the $y=x$ function, while the solid lines show $y=\gamma^{1 / 4} e^{-1 / 4 x^{2}}$ for different values of $\gamma$.

$$
\begin{aligned}
\mathcal{Z}_{\mathrm{dLr}}= & \lim _{B \rightarrow \infty} \frac{B}{\sqrt{\pi}} \int \mathcal{D} h(\mathbf{r}) \exp \left\{-\frac{\kappa}{2 T} \sum_{\mathbf{r}}\left\{[\Delta h(\mathbf{r})]^{2}\right.\right. \\
& \left.\left.+2 T B^{2} \sin ^{2} \pi h(\mathbf{r})\right\}\right\} .
\end{aligned}
$$

The sum in the exponent of Eq. (A4) is the Hamiltonian (2), with the identification $2 B^{2}=V_{0} / k_{B} T$. A completely analogous calculation relates the $\mathrm{dG}$ and $\mathrm{sG}$ models [22].

\section{APPENDIX B: SOLUTION OF THE SELF-CONSISTENT EQUATIONS}

In this Appendix we calculate the self-consistent solution of Eqs. (9) and (10) for the equilibrium correlation length of the variational approximation (3) to model (2). By defining $x=2 \kappa^{1 / 2} a_{\perp} T^{-1 / 2} \pi^{-1} \xi^{-1}$ and $\gamma=64 V_{0} a_{\perp}^{2} \kappa T^{-2} \pi^{-2}$, Eqs. (9) and (10) become, within the continuum approximation made in Sec. II,

$$
x^{4}=\gamma e^{-1 / x^{2}} .
$$

It is obvious that Eq. (B1) always has the solution $x=0$, and that for some values of $\gamma$ it may also have nonzero solutions. Our first aim is to determine the critical value of $\gamma$ for which $x=0$ is the unique solution. To this end, we rewrite the equation in the following way:

$$
x=\gamma^{1 / 4} e^{-1 / 4 x^{2}} .
$$

Now the solutions are the intersections of the function $y$ $=f(x)=\gamma^{1 / 4} e^{-1 / 4 x^{2}}$ with the straight line $y=x$. As we can see in Fig. 6, for $\gamma>\gamma_{C}$ there are three solutions of Eq. (B2), two solutions for $\gamma=\gamma_{C}$, and only the trivial solution $x=0$ for $\gamma<\gamma_{C}$. The value of $\gamma_{C}$ can be calculated using that for $\gamma=\gamma_{C}$ the unique solution $x=x_{s} \neq 0$ verifies Eq. (B2) and also the equation

$$
1=\gamma_{C}^{1 / 4} \frac{1}{2 x_{s}^{3}} e^{-1 / 4 x_{s}^{2}}
$$

obtained by requiring that the slopes of $y=x$ and $y=f(x)$ be equal at $x=x_{s}$. With these two equations it is easy to obtain $x_{s}=2^{-1 / 2}$ and $\gamma_{C}=e^{2} / 4$. Using the definition of $\gamma$, the temperature for which $\xi^{-1}=0$ is the only solution of Eq. (10) is then given by

$$
T_{C}=\frac{16 V_{0}^{1 / 2} \kappa^{1 / 2} a_{\perp}}{e \pi} .
$$

Now, for $T<T_{C}$ we have to determine which of the three solutions of Eq. (B1) provides the physical correlation length. Since $\xi^{-1}=0$ is the unique solution for high temperatures, we take as a reference value $\mathcal{F}_{V}\left(\xi^{-1}=0\right)$, and note that $\Delta \mathcal{F}(\xi)=\mathcal{F}_{V}(\xi)-\mathcal{F}_{V}\left(\xi^{-1}=0\right)$ is stationary at any root of Eq. (10). Thus, we will consider, as the physical solution for the correlation length, that root of Eq. (10) for which $\mathcal{F}_{V}$ has an absolute minimum. Starting out with high temperatures, the condition $\Delta \mathcal{F}_{V}(\xi)=0$ will signal the temperature at (and below) which $\xi^{-1}=0$ ceases to be the global minimum of the variational free energy and thus the system physical correlation length. Using our previous notation, the condition $\Delta \mathcal{F}_{V}(\xi)=0$ reads

$$
x^{2}=\gamma^{\prime} e^{-1 / x^{2}},
$$

where $\gamma^{\prime}=64 \kappa a_{\perp}^{2} V_{0} /\left(\pi^{2} T^{2}\right)$. Using the same argument as above, it is easy to show that for $\gamma^{\prime}<\gamma_{R}^{\prime}=e$ there are nonzero solutions of Eq. (B5). This means, using the definition of $\gamma^{\prime}$, that there is a temperature given by

$$
T_{R}=\frac{e^{1 / 2}}{2} T_{C},
$$

such that for $T<T_{R}$ the global minimum of the free energy is attained for a correlation length $\xi \neq 0$, whereas for $T \geqslant T_{R}$ the physical solution is $\xi_{\text {phys }}^{-1}=0$.

\section{APPENDIX C: TRIANGULAR LATTICE}

The Laplacian roughening model was initially proposed by Nelson on the triangular lattice [13]. Thus, it is worth studying how do the features of our model (2) change when the substrate geometry is different from the square lattice considered in the text. Nevertheless, we expect that only nonuniversal quantities - such as the transition temperature and the numerical value of correlation length-depend upon the lattice geometry. The Laplacian roughening model on the triangular lattice is given by

$$
\mathcal{H}_{\mathrm{LR}}=\frac{\kappa}{2} \sum_{i}\left[\sum_{\delta}\left(h_{i}-h_{i+\delta}\right)\right]^{2}
$$

with $i+\delta$ being any of the six nearest neighbors of site $i$. For this case [25], $\quad \omega(\mathbf{q})=16\left\{\sin ^{2}\left(q_{x} / 2\right)+\sin ^{2}\left[\left(q_{x}+\sqrt{3} q_{y}\right) / 4\right]\right.$ $\left.+\sin ^{2}\left[\left(q_{x}-\sqrt{3} q_{y}\right) / 4\right]\right\}^{2}$, where $\mathbf{q}=\left(n_{x} / L\right) \mathbf{b}_{x}+\left(n_{y} / L\right) \mathbf{b}_{y}$, with $\mathbf{b}_{x}=2 \pi\left[\mathbf{e}_{x}-(1 / \sqrt{3}) \mathbf{e}_{y}\right]$ and $\mathbf{b}_{y}=(4 \pi / \sqrt{3}) \mathbf{e}_{y}$, where $n_{i}$ $=-(L-1) / 2, \ldots, L / 2$ and $\mathbf{e}_{i}$ are the standard basis vectors. In the continuum limit, $S_{0}(\mathbf{q}) \simeq 4 T /\left(9 \kappa \mathbf{q}^{4}\right)$, and we recover Eq. (10). Taking the continuum limit, i.e, 


$$
\frac{1}{L^{2}} \sum_{\mathbf{q}} \rightarrow \frac{\sqrt{3}}{2} \int_{\operatorname{BZ}(2 \pi)^{2}} \simeq \frac{d^{2} \mathbf{q}}{4 \pi} \int_{0}^{(2 / \sqrt{3})^{1 / 2} \pi} q d q,
$$

where BZ denotes the first Brillouin zone, we get

$$
w^{2} \simeq \frac{T}{2 \pi \kappa(2 / \sqrt{3})^{2}} \int_{0}^{\pi} \frac{q^{\prime}}{q^{\prime 4}+\xi^{-4} /(2 / \sqrt{3})^{2}} d q^{\prime} .
$$

Thus, by defining $T^{\prime}=T / 3$, and $\xi^{\prime}=3^{1 / 4} \xi$, we get the same Eq. (12) but with redefined constants $T^{\prime}$ and $\xi^{\prime}$. One can readily reproduce all the results obtained in the text, simply by making the replacements $T \rightarrow T^{\prime}$ and $\xi \rightarrow \xi^{\prime}$. In conclusion, on the triangular lattice a first-order roughening transition is also obtained, the only effect of the geometry being a shift in the value of the roughening temperature $T_{R}^{\text {triang }}$ $=3 T_{R}^{\text {square }}$.

\section{APPENDIX D: SUBSTRATE DIMENSIONS $d \neq 2$}

In this Appendix we discuss the possibility to find a roughening transition in equilibrium when model (2) is defined on a substrate of generic dimension $d$. In such a case Eq. (10) is still valid, but with

$$
w^{2}(\xi) \simeq \int \frac{d^{d} \mathbf{q}}{(2 \pi)^{d}} \frac{T}{\kappa\left[\omega(\mathbf{q})+\xi^{-4}\right]}
$$

within the continuum limit. For substrate dimension $d>4$, the integral (D1) is finite for $\xi^{-1}=0$, namely, $w^{2}\left(\xi^{-1}=0\right)$ $=K_{d} \pi^{d-4} T /[\kappa(d-4)]$ (where $K_{d}$ is the $d$-dimensional angular integral $\left.K_{d}=\int d^{d-1} \Omega /(2 \pi)^{d}=2 \pi^{d / 2} /\left[(2 \pi)^{d} \Gamma(d / 2)\right]\right)$. Thus, $\xi^{-1}=0$ is no longer a solution of Eq. (10). Therefore the system has no rough solution and is in the flat phase for all temperatures. On the other hand, for $d<4$, the integral above may be approximated by

$$
w^{2}(\xi) \lesssim \frac{T}{\kappa} \frac{\pi K_{d}}{4 \sin (\pi d / 4)} \xi^{4-d} .
$$

In this case, $\xi^{-1}=0$ is always solution of Eq. (10), there being two additional finite solutions when $T<T_{C}^{d}$. The value of $T_{C}^{d}$ can be calculated using the same argument as in $d$ $=2$ and is

$$
T_{C}^{d<4}=\frac{8 \kappa a_{\perp}^{2} \sin (d \pi / 4)}{(4-d) K_{d} e \pi^{3}}\left(\frac{4 \pi^{2} V_{0}}{\kappa a_{\perp}^{2}}\right)^{1-d / 4} .
$$

In order to know which solution of Eq. (10) minimizes the variational free energy, we calculate $\Delta \mathcal{F}_{V}$, which now reads

$$
\frac{\Delta \mathcal{F}_{V}(\xi)}{T L^{d}} \simeq \frac{4-d}{d} \frac{\pi K_{d}}{8 \sin (\pi d / 4)} \xi^{-d}-\frac{V_{0}}{T} e^{-2 \pi^{2} w^{2} / a_{\perp}^{2}}
$$

In this case, the local minimum $\xi_{2}^{-1} \neq 0$ is also the global minimum and the physical solution for temperatures below the roughening temperature $\left(T<T_{R}^{d<4}\right)$, which is given by

$$
T_{R}^{d<4}=\frac{d}{4} e^{1-d / 4} T_{C}^{d<4}
$$

For temperatures above roughening $\left(T \geqslant T_{R}^{d<4}\right), \xi^{-1}=0$ provides the global free-energy minimum. Thus, for $d<4$ there is a first-order roughening transition at $T_{R}^{d<4}$. Note this includes $d=1$, which might seem conflictive since in this case model (2) is expected to be in the rough phase for all values of $T$ [13]: In $d=1$ thermal fluctuations are expected to destroy the ordered flat phase for any temperature value. Our result can be understood by noting that $\mathcal{F}_{V}$ is not a true free energy, in the sense that it is not the free energy of any model [32], but rather an upper bound for the free energy of model (2). Actually, exactly the same result is obtained in the variational study of the $\mathrm{sG}$ model in $d=1$ [5]. Note that in this reference the analysis of the $d=1$ case is incomplete, with the incorrect conclusion that the variational theory predicts no phase transition when $d=1$. The complete expression for $\Delta \mathcal{F}_{V}(\xi)$ analogous to Eq. (D4) indeed shows that for the $\mathrm{sG}$ model in $d=1$, the variational approximation predicts a nonzero temperature below which the physical value of the correlation length is finite.

Finally, for $d=4$, Eq. (10) is very similar to that obtained by Saito [5] for the sine-Gordon model

$$
\kappa \xi^{-4}=\frac{4 \pi^{2} V_{0}}{a_{\perp}^{2}}\left(1+\pi^{4} \xi^{4}\right)^{-\left(T / 16 \kappa a_{\perp}^{2}\right)} .
$$

Following Saito's analysis, we readily obtain that for $d=4$ our model has a Kosterlitz-Thouless transition at $T=T_{R}^{d=4}$ $\equiv 16 \kappa a_{\perp}^{2}$. The correlation length now diverges as $\xi \sim \exp$ $\left\{-A /\left(T-T_{R}^{d=4}\right)\right\}$ when $T \rightarrow T_{R}^{d=4,-}$ ( $A$ being a $T$ independent constant).

In summary, within the variational approach, our model displays a first-order transition for $d<4$ between a flat phase and a rough phase with the properties of the linear MBE equation. For the marginal dimension $d=4$ this transition becomes of the Kosterlitz-Thouless type, whereas for $d>4$ the surface is in the flat phase for all temperature values.
[1] A.-L. Barabási and H. E. Stanley, Fractal Concepts in Surface Growth (Cambridge University Press, Cambridge, England, 1995).

[2] Y. Saito, Statistical Physics of Crystal Growth (World Scientific, Singapore, 1996)
[3] J.D. Weeks, in Ordering in Strongly Fluctuating Condensed Matter Systems, edited by T. Riste (Plenum, New York, 1980).

[4] J. Lapujolade, Surf. Sci. Rep. 20, 191 (1994).

[5] Y. Saito, Z. Phys. B: Condens. Matter 32, 75 (1978).

[6] P. Nozières and F. Gallet, J. Phys. (France) 48, 353 (1987). 
[7] A. Sánchez, D. Cai, N. Grønbech-Jensen, A.R. Bishop, and Z.J. Wang, Phys. Rev. B 51, 14664 (1995).

[8] M. Kotrla and A.C. Levi, Surf. Sci. 317, 183 (1994).

[9] A. Diehl, M.C. Barbosa, and Y. Levin, Phys. Rev. E 56, 619 (1997).

[10] A. Hazareesing and J.-P. Bouchaud, Eur. Phys. J. B 14, 713 (2000).

[11] A.N. Berker and D.R. Nelson, Phys. Rev. B 19, 2488 (1979).

[12] S.A. Safran, Statistical Thermodynamics of Surfaces, Interfaces, and Membranes (Addison Wesley, Reading, MA, 1994).

[13] D.R. Nelson, Phys. Rev. B 26, 269 (1982).

[14] K.J. Strandburg, Rev. Mod. Phys. 60, 161 (1988).

[15] W. Janke and H. Kleinert, Phys. Lett. A 114, 255 (1986).

[16] W. Janke and H. Kleinert, Phys. Rev. B 41, 6848 (1990).

[17] W. Janke and D. Toussaint, Phys. Lett. A 116, 387 (1986).

[18] J.G. Dash, Rev. Mod. Phys. 71, 1737 (1999).

[19] E. Moro, R. Cuerno, and A. Sánchez, Phys. Rev. Lett. 78, 4982 (1997).

[20] E. Moro, Ph.D. thesis, Universidad Carlos III de Madrid, 1999 (unpublished) (in Spanish); http://www-thphys.physics.ox.ac. uk/users/EstebanMoro/profesional html

[21] J.J. Ruiz-Lorenzo, E. Moro, R. Cuerno, and A. Sánchez (un- published)

[22] H. Müller-Krumbhaar, in 1976 Crystal Growth and Materials, edited by E. Kaldis and H.J. Scheel (North-Holland, New York, 1977).

[23] Y. Levin and K.A. Dawson, Phys. Rev. A 42, 3507 (1990).

[24] In Ref. [5] it was obtained that, for $T \rightarrow T_{R}^{s G,-}$, the correlation length behaves as $\xi \sim \exp \left\{C\left(T_{R}^{s G}-T\right)^{-1}\right\}$, whereas more accurate calculations conclude $\xi \sim \exp \left\{C\left(T_{R}^{s G}-T\right)^{-1 / 2}\right\}$. Here $C$ is a $T$-independent constant.

[25] S. Prestipino and E. Tosatti, Phys. Rev. B 59, 3108 (1999).

[26] J.-P. Bouchaud and A. Georges, Phys. Rev. Lett. 68, 3908 (1992).

[27] We make the homogeneity assumption that $\left\langle h_{i}(t)\right\rangle$ is independent of substrate position, see Ref. [5].

[28] H.-N. Yang, G.-C. Wang, and T.-M. Lu, Phys. Rev. B 51, 17 932 (1995).

[29] D.A. Bruce, Mater. Sci. Forum 4, 51 (1985).

[30] K.J. Strandburg, S.A. Solla, and G.V. Chester, Phys. Rev. B 28, 2717 (1983).

[31] R. Cuerno and E. Moro (unpublished).

[32] The free energy of model $\mathcal{H}_{0}$ is $\mathcal{F}_{0}$. 